\title{
Optimizing Strategy for the Dielectric Performance of Topological-structured Polymer Nanocomposites by Rationally Tailoring the Spatial Distribution of Nanofillers
}

\author{
Liang Sun, ${ }^{1}$ Liang Liang, ${ }^{1}$ Zhicheng Shi,,${ }^{1, *}$ Huanlei Wang, ${ }^{1}$ Peitao Xie, ${ }^{2, *}$ Davoud Dastan, ${ }^{3}$ Kai Sun ${ }^{4}$ and Runhua Fan ${ }^{4}$
}

\begin{abstract}
There is a mindset that functional fillers tend to be as homogeneous as possible to achieve high dielectric constant and low loss when designing polymer dielectric composites. This study breaks the mindset by proposing a novel inhomogenous design in a topological-structured polymer nanocomposite, the dielectric performance is optimized by rationally controlling the spatial distribution of nanofillers. We prepared layered composites where $\mathrm{BaTiO}_{3}$ and $\mathrm{TiN}^{\mathrm{N}}$ nanoparticles were dispersed in the top and bottom section of the poly(vinylidene difluoride-co-hexafluoropropylene) (P(VDF-HFP)) composites, respectively. Interestingly, the layered inhomogenous composites exhibit obviously enhanced dielectric permittivity (1.5 times as high), suppressed loss (a $70 \%$ drop) and improved breakdown strengths (4.2 times as high) compared with their single-layer homogenous counterparts. This dielectric optimization is mainly attributed to the design of multilevel interfaces and the redistribution of polarization charges in the inhomogenous topological structure. This research provides a novel optimizing strategy and will promote the development of dielectrics.
\end{abstract}

Keywords: Polymer composites; Dielectric property; Multilayer structure; Film capacitor; Interfacial polarization.

Received: 31 August 2020; Accepted: 31 October 2020.

Article type: Research article

\section{Introduction}

High dielectric constant (high- $k$ ) materials have gained everincreasing attention in recent years because of their wide applications in capacitors, ${ }^{[1-6]}$ actuators, ${ }^{[7-10]}$ microstrip antennas, ${ }^{[11,12]}$ field-effect transistors (FETs), ${ }^{[13,14]}$ and high voltage transmission lines, ${ }^{[15,16]}$ etc. So far, various strategies have been developed to achieve improved dielectric performances (i.e., high dielectric constant, low loss, and high breakdown strength, etc.), among which constructing polymer composites filled with conductive fillers (e.g., metals, carbon nanotubes, graphene, conductive polymers, etc ${ }^{[12,17-25]}$ or

\footnotetext{
${ }^{1}$ School of Materials Science and Engineering, Ocean University of China, Qingdao 266100, ChinaA

${ }^{2}$ State Key Laboratory of Bio-fibers and Eco-textiles, Institute of Biochemical Engineering, College of Materials Science and Engineering, Qingdao University, Qingdao 266071, China

${ }^{3}$ Department of Materials Science and Engineering, Georgia Institute of Technology, Atlanta, Georgia-30332, USA

${ }^{4}$ Institute of Marine Materials Science and Engineering, Shanghai Maritime University, Shanghai 201306, China.

*Email: zcshi@ouc.edu.cn (Z. Shi); xiepeitao1991@qdu.edu.cn (P. Xie)
}

ferroelectric ceramic (e.g., $\mathrm{BaTiO}_{3}, \mathrm{SrTiO}_{3}, \mathrm{CaCu}_{3} \mathrm{Ti}_{4} \mathrm{O}_{12}$ (CCTO), etc) ${ }^{[26-32]}$ is believed to be an effective way to achieve high-performance dielectrics. These two typical strategies always pursue a homogeneous distribution of functional fillers as far as possible for the aim of simultaneous high dielectric constant and low loss. However, these two typical strategies still have a long way to go for the goal. For instance, although ultrahigh dielectric constants over $10^{3} @ 1 \mathrm{kHz}$ have been achieved in percolative conductor/polymer composites, severely elevated loss along with sharply decreased breakdown strengths are always inevitable. As for the ferroelectric ceramic/polymer composites, the enhancement of dielectric constant is usually far below expectations even the loading fractions of the ferroelectric ceramic fillers exceed 50 vol\%, and breakdown strengths are obviously deteriorated. ${ }^{\text {[33- }}$ 35] Consequently, the achievement of enhanced dielectric constant without sacrificing low loss and high breakdown strength is a challengeable problem to be addressed until now.

Considering the inherent limitations of the aforementioned two types of high- $k$ polymer composites, researchers adopted 
(a)

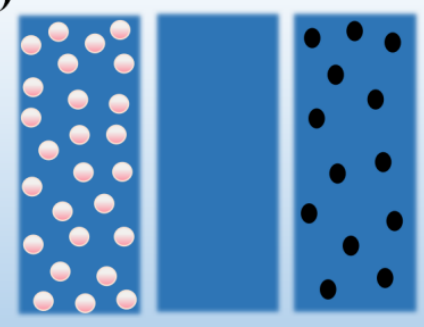

respective single-layer

Pure PVDF (b)

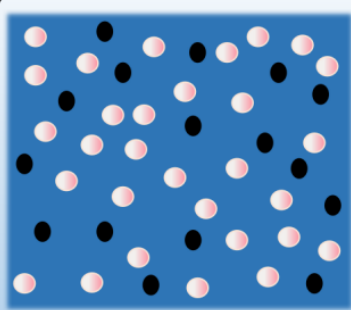

ternary single-layer (c)

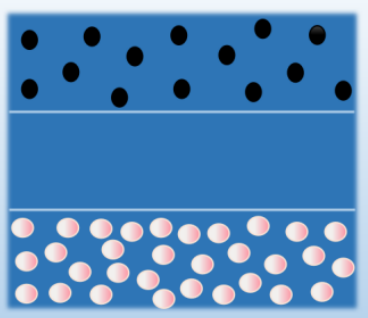

trilayered composite

Fig. 1 The schematic spatial distribution of barium titanate and titanium nitride particles in the respective single-layer, ternary single-layer and ternary trilayer composites.

the ternary polymer composites co-filled with ferroelectric ceramics and conductors in order to effectively balance the dielectric constant, loss, and breakdown strength. Following this idea, various ternary polymer composites were designed and the dielectric performances were explored. For example, Wang et al. fabricated the ferroferric oxide/multiwalled carbon nanotube (MWNT)/polyvinylidene fluoride (PVDF) composites, ${ }^{[36]}$ whose dielectric constant was significantly improved while the loss remained at a lower level, compared with their binary counterparts of $\mathrm{Fe}_{3} \mathrm{O}_{4}$ /PVDF and MWNT$\mathrm{s} / \mathrm{PVDF}$. Hu et al. designed the polypropylene (PP) composites co-filled with semi-conductive tetra-needle- shaped zinc oxide $(\mathrm{ZnO})$ whiskers and barium titanate (BT) nanoparticles, ${ }^{[37]}$ where an improvement of dielectric permittivity (from 8.5 to 11) and a decline of loss tangent (from 0.008 to 0.004 ) could be realized simultaneously. Jaschin et al. constructed a ternary percolative polymer composite consisting of lanthanum nickelate $\left(\mathrm{LaNiO}_{3}\right)$ nanocrystals, barium titanate $\left(\mathrm{BaTiO}_{3}\right)$ nanocrystals and PVDF, ${ }^{[38]}$ a high dielectric constant of 90 at $10 \mathrm{kHz}$ (and a low loss of 0.13 ) was obtained with the $\mathrm{LaNiO}_{3}$ content below the percolation threshold. The achieved dielectric constant is around 9 times that of pure PVDF and 3 times that of $\mathrm{BaTiO}_{3} / \mathrm{PVDF}$ nanocomposite. Up to now, extensive researches of constructing ternary composites with excellent comprehensive dielectric performances have been carried out and many encouraging progresses have been made. However, the improvement is still not satisfactory, and the design of the ternary composites still follows the principles that the nanofillers should be homogeneous in the polymer matrix to obtain low dielectric loss as well as high dielectric constant. ${ }^{[39-45]}$

In this paper, we proposed a novel inhomogenous design in a layered topological-structured polymer nanocomposite, in which the ferroelectric ceramic fillers and conductive fillers were restricted in respectively separated layers, and the dielectric performance was adjusted by rationally controlling the spatial distribution of these nanofillers, as shown in Fig. 1. It is demonstrated that, the layered inhomogenous composites exhibited enhanced dielectric constants, suppressed loss and obviously improved breakdown strengths in comparison with their single-layer homogenous ternary counterparts. Furthermore, the layered composites also displayed much higher energy densities and efficiencies than that of the corresponding single-layer composites.

\section{Experimental}

\subsection{Materials}

Barium titanate $\left(\mathrm{BaTiO}_{3},<300 \mathrm{~nm},>99.5 \%\right.$, Aladdin Industrial Corporation), titanium nitride (TiN, 20 nm, $99.9 \%$, Shanghai Macklin Biochemical Co., Ltd.), poly (vinylidene fluoride-hexafluoropropylene) (P(VDF-HFP), $15 \%$ HFP, PolyK Technologies, USA), 1-Methyl-2-pyrrolidone (NMP, $\mathrm{C}_{5} \mathrm{H}_{9} \mathrm{NO}, \geq 99.0 \%$ Sinopharm Chemical Reagent Co., Ltd.), sodium dodecylbenzenesulfonate $\left(\mathrm{C}_{18} \mathrm{H}_{29} \mathrm{NaO}_{3}, \geq 88.0 \%\right.$, Sinopharm Chemical Reagent Co., Ltd.) and ethanol ( $\geq 99.7 \%$, Sinopharm Chemical Reagent Co., Ltd.) were purchased and used without any further treatment.

\subsection{Preparation of single-layer and trilayer composites}

Pure $\mathrm{P}(\mathrm{VDF}-\mathrm{HFP})$, single-layer $\mathrm{BaTiO}_{3} / \mathrm{P}(\mathrm{VDF}-\mathrm{HFP})$ and TiN/P(VDF-HFP) composites were prepared via solution casting and subsequent hot-pressing techniques. Firstly, $\mathrm{BaTiO}_{3}$, TiN and sodium dodecylbenzenesulfonate (SDBS) were ultrasonically dispersed into $10 \mathrm{~mL} \mathrm{NMP} \mathrm{for} 1 \mathrm{~h}$ to obtain a stable suspension at room temperature. Then, P(VDFHFP) pellets were added into the suspension under magnetic stirring at $75^{\circ} \mathrm{C}$ until they were dissolved completely. For the preparation of pure PVDF solution, the P(VDF-HFP) pellets were directly dissolved in NMP under rapid magnetic stirring at $75{ }^{\circ} \mathrm{C}$ for $5 \mathrm{~h}$ and slow stirring at room temperature overnight. The solution casting method was adopted to prepare the single-layer composites. Specifically, the as prepared solution was casted on a glass plate followed by drying in an oven at $100{ }^{\circ} \mathrm{C}$ for $4 \mathrm{~h}$ and $200^{\circ} \mathrm{C}$ for $5 \mathrm{~min}$. The films were peeled off via quenching in ice water and dried in an oven at $70{ }^{\circ} \mathrm{C}$ for $6 \mathrm{~h}$, forming the single-layer BT/P(VDF-HFP), TiN/P(VDF-HFP) and pure P(VDF-HFP) films. Finally, trilayered films were obtained via stacking and hot-pressing a BT/P(VDF-HFP) film, a pure P(VDF-HFP) film and a TiN/P(VDF-HFP) film at $160^{\circ} \mathrm{C}$ under a pressure of $8 \mathrm{MPa}$ 
for $20 \mathrm{~min}$. The thicknesses of the single-layer composites were controlled in the range of $10-15 \mu \mathrm{m}$ via adjusting the height of the blade. The thicknesses of the trilayer composite films were controlled to be $35-40 \mu \mathrm{m}$. For example, the sample code of the ternary composites with one or three layers can be expressed as 10-0.3, the meaning is that the volume fractions of $\mathrm{BaTiO}_{3}$ and $\mathrm{TiN}$ in the ternary composites are 10 and 0.3 vol $\%$, respectively.

\subsection{Morphology and composition characterization}

The morphologies of the trilayer films and the elemental distributions of barium, titanium, nitrogen and carbon were observed by scanning electron microscopy (SEM, S-4800, Hitachi, Ltd.) coupled with nergy-dispersive X-ray spectroscopy (EDX). The compositions of the trilayered composites were analyzed by X-ray diffractometer (XRD, D8, Advance, Bruker, Ltd.).

\subsection{Dielectric spectra, breakdown strength and energy- storage measurements}

For the dielectric measurement, circular gold electrodes with a diameter of $2.98 \mathrm{~mm}$ were sputtered on both sides of the samples before dielectric measurements. The dielectric properties were analyzed with an Agilent E4980A Precision LCR analyzer in the frequency range from $100 \mathrm{~Hz}$ to $1 \mathrm{MHz}$. Open and short compensations were performed before testing. The permittivity was calculated by $\varepsilon_{\mathrm{r}}=t C_{\mathrm{p}} / A \varepsilon_{0}$, where $t$ is the thickness of the sample, $A$ is the area of the electrode, $C_{\mathrm{p}}$ is the parallel capacitance, and $\varepsilon_{0}$ is the absolute permittivity of free space $\left(8.85 \times 10^{-12} \mathrm{~F} \mathrm{~m}^{-1}\right)$. The breakdown strengths were obtained using a setup equipped with a Treck 609A amplifier with a voltage ramping rate of $500 \mathrm{~V} / \mathrm{s}$ at room temperature (PolyK Technologies, USA). The energy storage performances, including discharge energy densities and charge-discharge efficiencies, were derived from the $P-E$ loops, where $P$ is the electric displacement, $E$ is the electric field. The loops were collected at $1 \mathrm{kHz}$ using a ferroelectric test system based on a modified Sawyer-Tower circuit at room temperature (PolyK Technologies, USA).

\section{Results and discussions}

The optical photograph and cross-sectional SEM of the 10-1 trilayer composite are presented in Fig. 2. As seen, the trilayer film is flexible and has smooth surfaces. The SEM image confirms the distinct trilayer configuration, in which the three layers are tightly welded together without the appearance of noticeable defects (e.g., pores, cracks, etc.). Moreover, the high-magnification cross-sectional SEM images shown in Fig. $\mathrm{S} 1$ demonstrate that the $\mathrm{BaTiO}_{3}$ particles are homogeneously dispersed in the P(VDF-HFP) matrix. The EDX mapping results further demonstrate that the $\mathrm{BaTiO}_{3}$ and $\mathrm{TiN}$ nanoparticles are well restricted in the P(VDF-HFP) matrix without any apparent diffusion into the middle P(VDF-HFP) layer, as shown in Fig. 2 and Fig. S1. In this work, the thicknesses of the single layer and multilayer composites were well controlled via adjusting the scraper height and the hotpressing parameters.

Fig. 2e shows the XRD patterns of the trilayer composite consisting of a pure $\mathrm{P}(\mathrm{VDF}-\mathrm{HFP})$ layer sandwiched by a TiN/ $\mathrm{P}(\mathrm{VDF}-\mathrm{HFP})$ composite layer with $1 \mathrm{vol} \%$ TiN content and a BT/P(VDF-HFP) composite layer with 10 vol\% BT content. The characteristic diffraction peaks corresponding to the (1 0 $0),\left(\begin{array}{lll}1 & 1 & 0\end{array}\right)$ and $\left(\begin{array}{lll}1 & 1 & 1\end{array}\right)$ crystal planes of perovskite $\mathrm{BaTiO}_{3}$ are observed at $2 \theta=22.0^{\circ}, 31.4^{\circ}$ and $38.7^{\circ}$, respectively. Only one weak characteristic diffraction peak of TiN is observed at $2 \theta=$ $74.068^{\circ}$ because of the ultralow filling fraction of TiN nanoparticles (in Fig. S1b). No additional diffraction peaks are observed, indicating that no impurities were introduced into the composite during the preparation process.
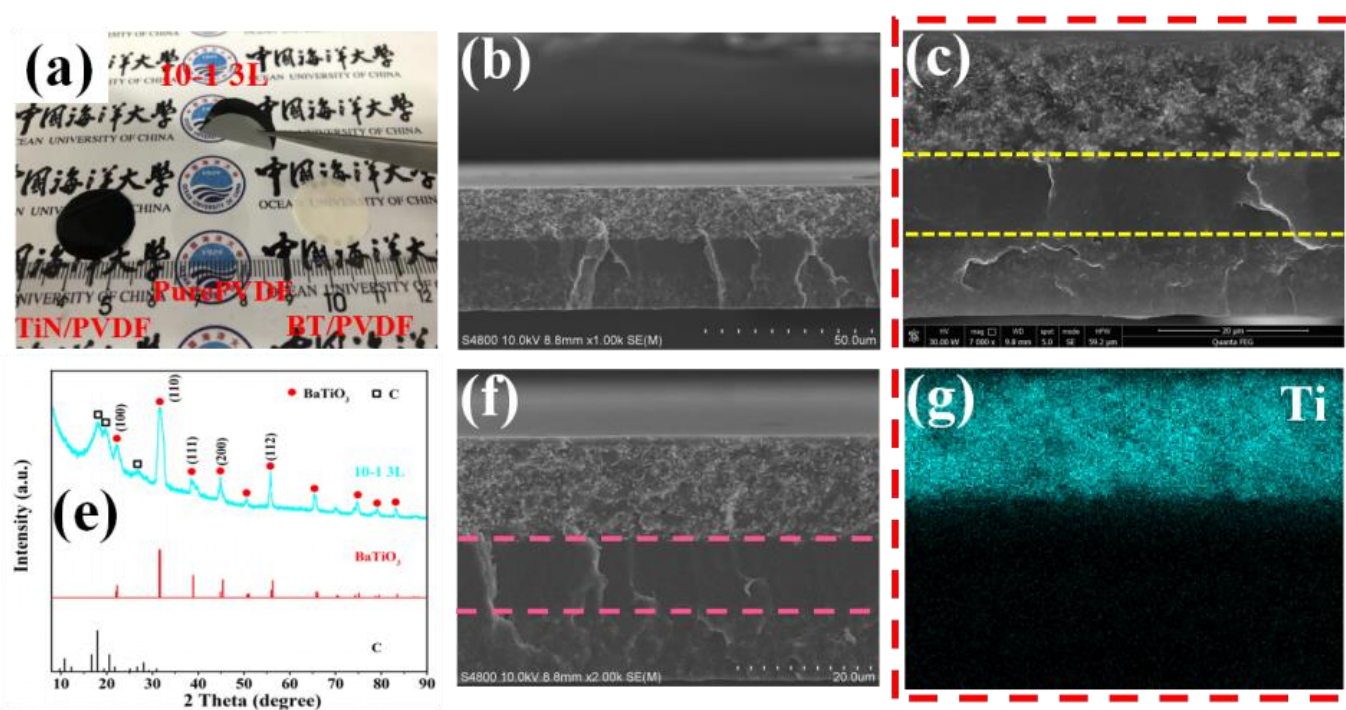
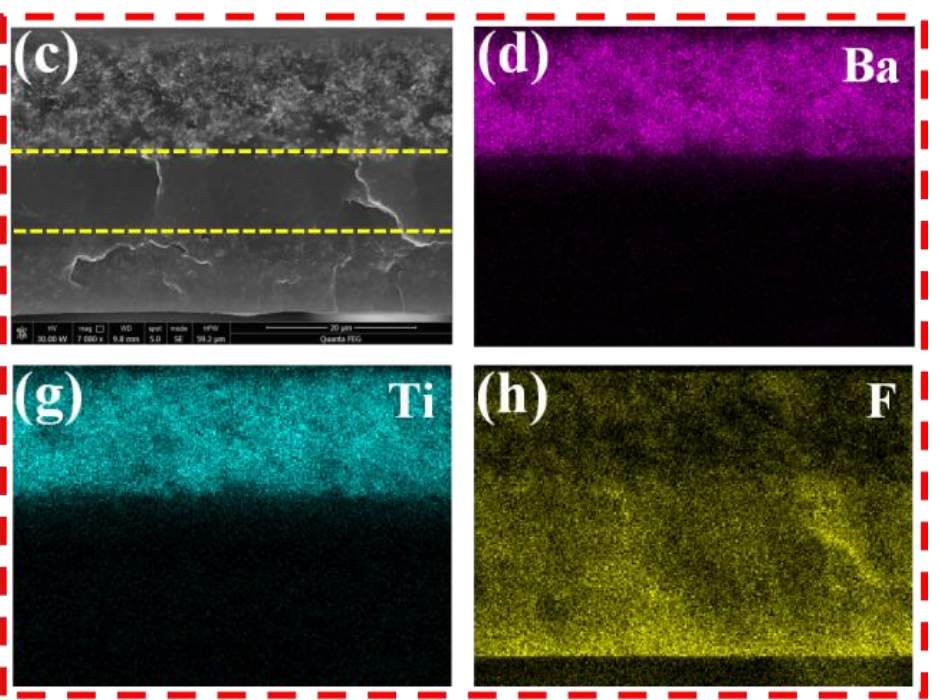

Fig. 2 (a) Photographs of the single-layer and trilyer composites. (b-d and f-h) Cross-sectional SEM morphologies and corresponding EDS mapping images of the trilayer composites.(e) XRD patterns of trilayer composite. 
(a)

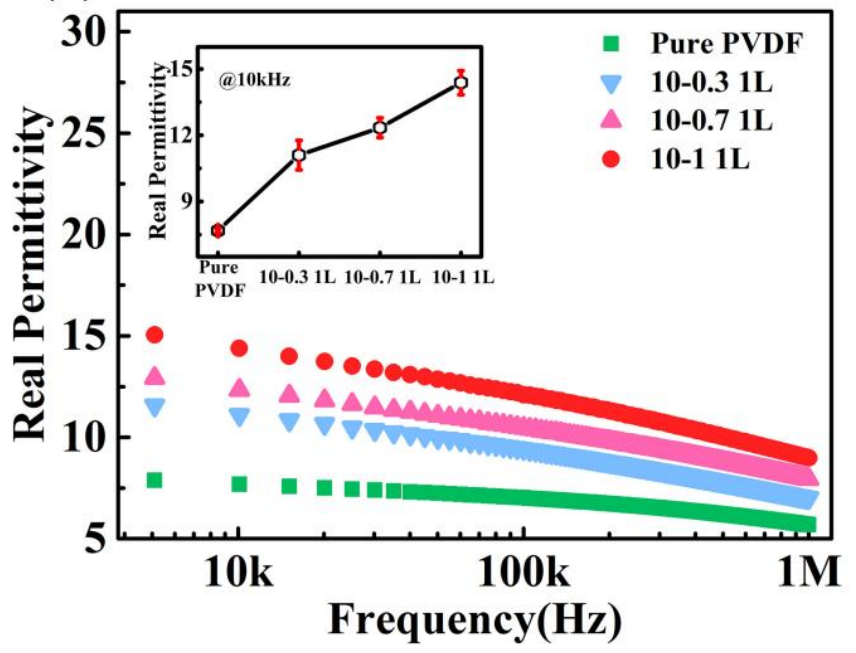

(c)

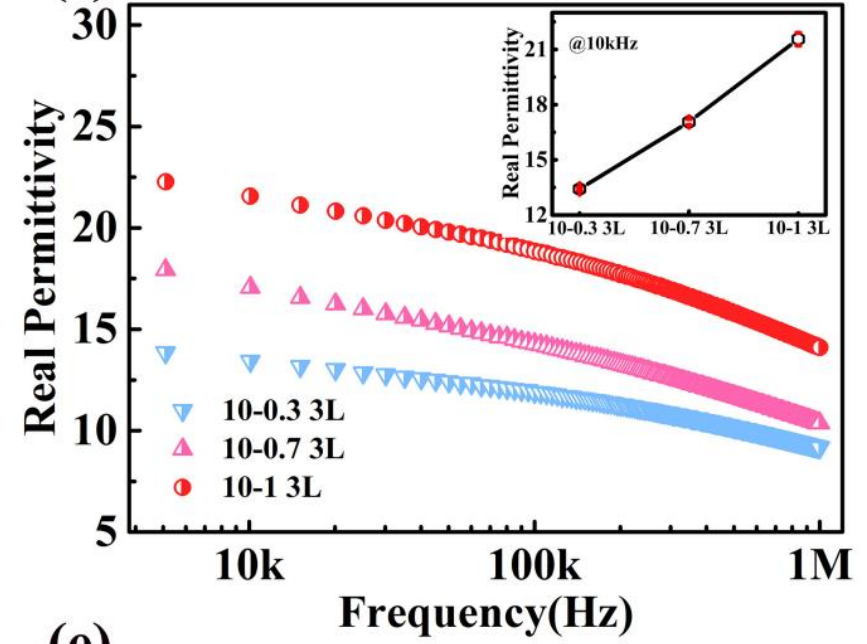

(e)

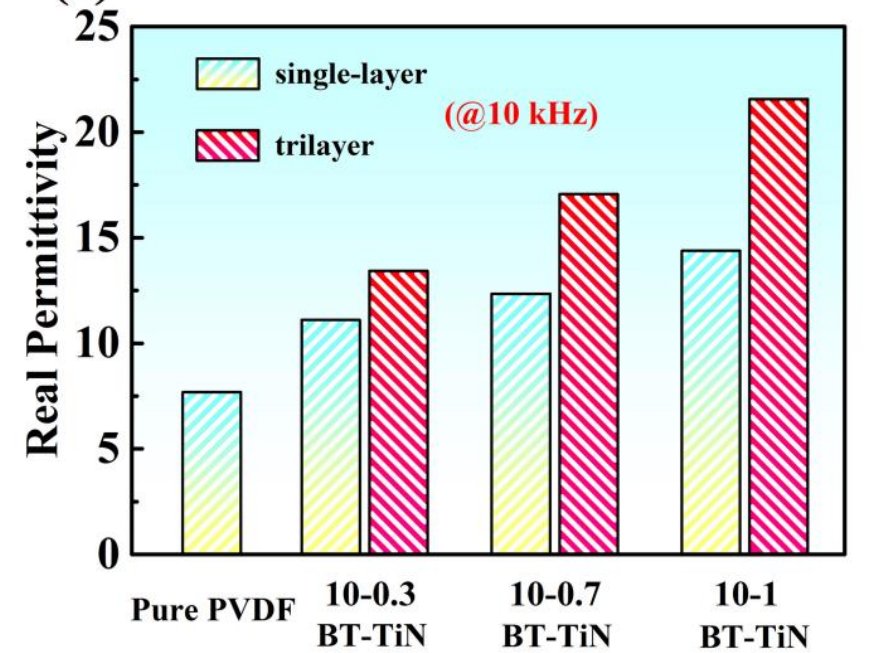

(b)

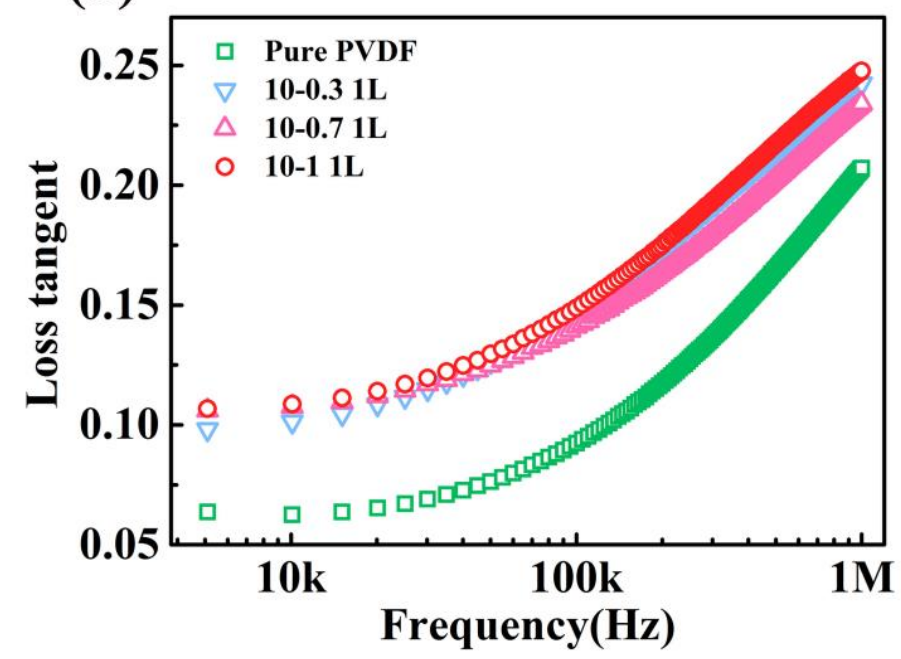

(d)

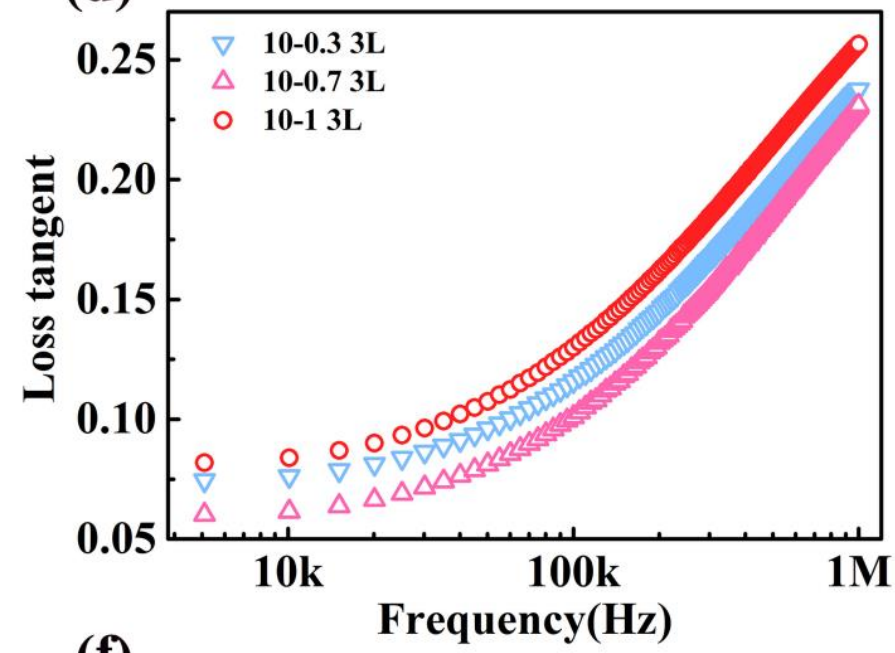

(f)

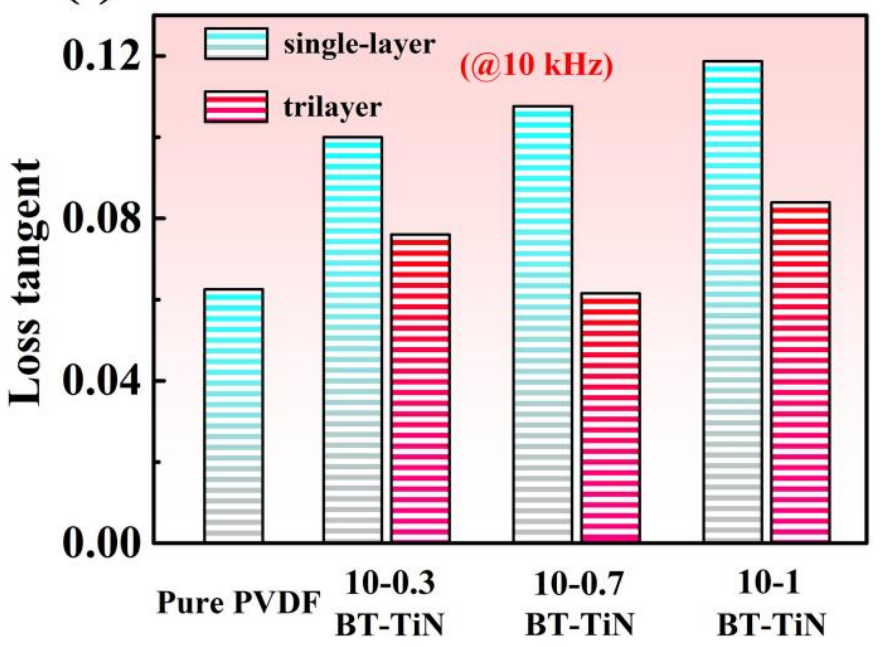

Fig. 3 Frequency dependences of dielectric permittivity and loss tangents for (a, b) single-layer and (c, d) trilayer BT/TiN/PVDF composites. $(\mathrm{e}, \mathrm{f})$ The comparison of dielectric performance at $10 \mathrm{kHz}$ between the hybrid single-layer and trilayer composites with the same content of $\mathrm{TiN}$ and $\mathrm{BaTiO}_{3}$. 

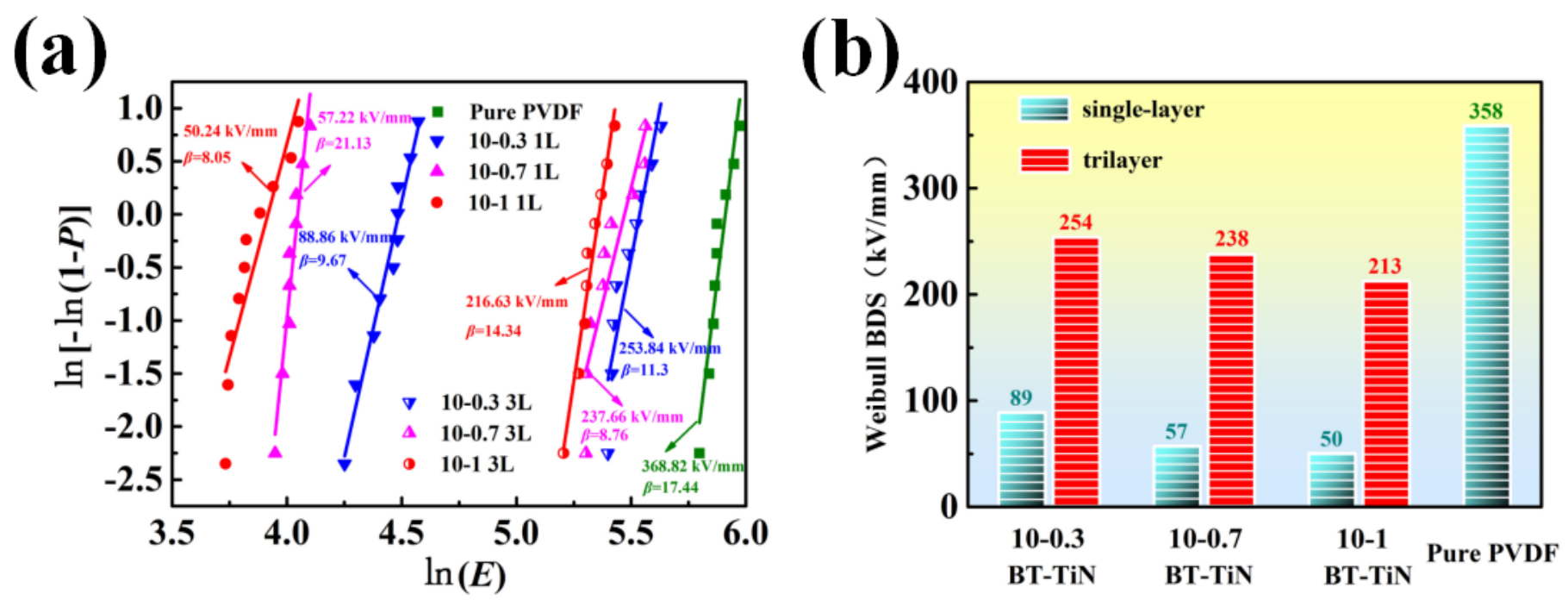

Fig. 4 (a) Weibull distribution plots, (b) the comparison of characteristic breakdown strengths between the hybrid single-layer and trilayer composites with the same content of $\mathrm{TiN}$ and $\mathrm{BaTiO}_{3}$.

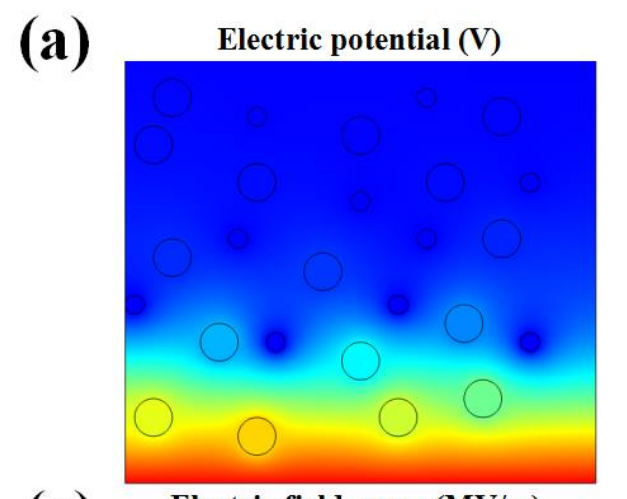

(c)

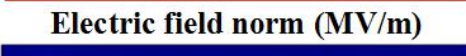

(e)
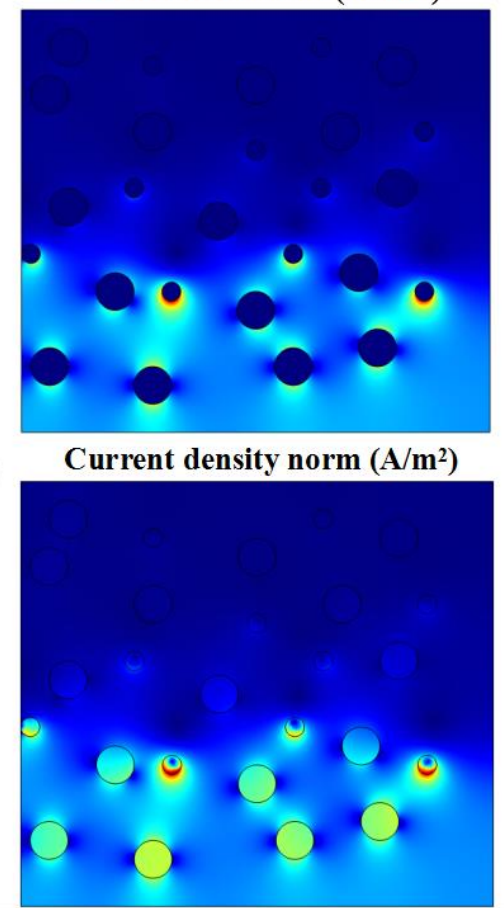

(b)

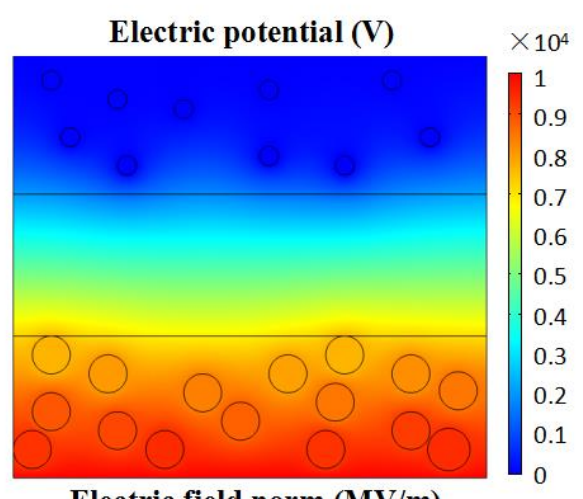

(d)

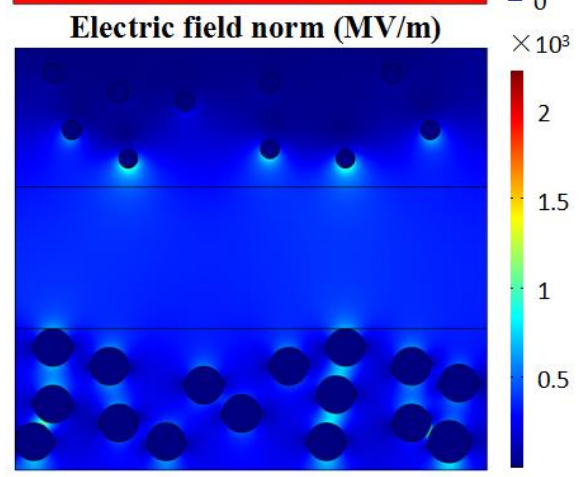

(f)

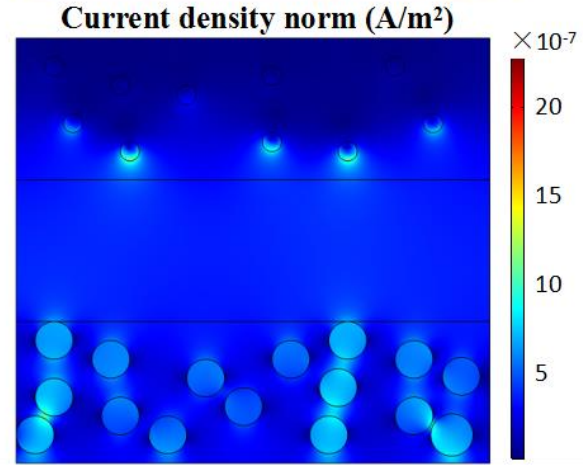

Fig. 5 The distribution of (a,b) electric potential, $(c, d)$ electric field strength and (e,f) leakage current density in single-layer and trilayer composites by finite element simulation. 
(a)

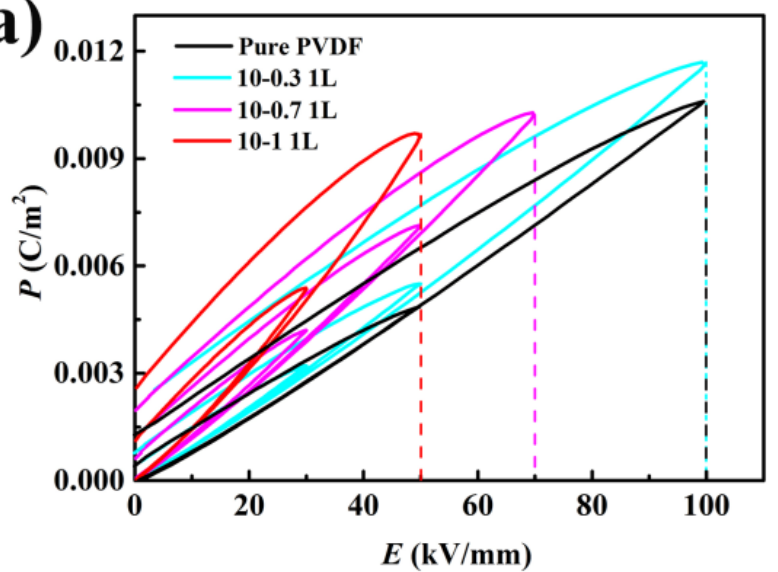

(c)

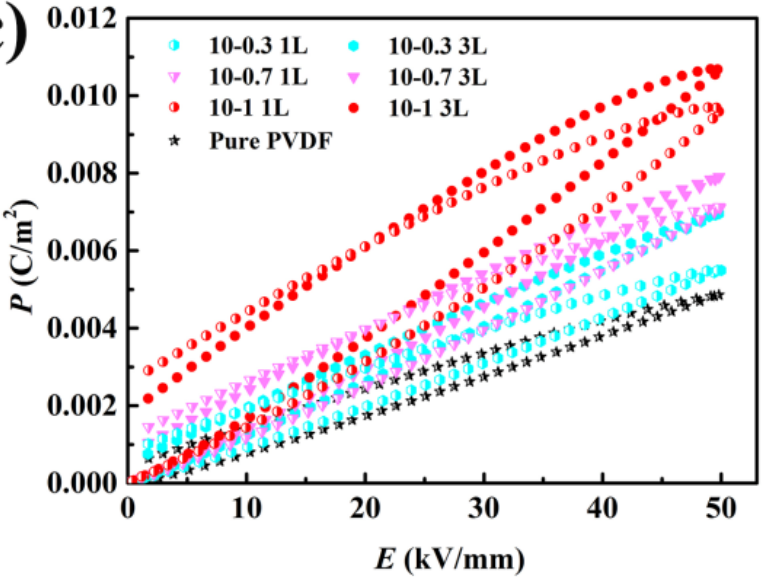

(e)

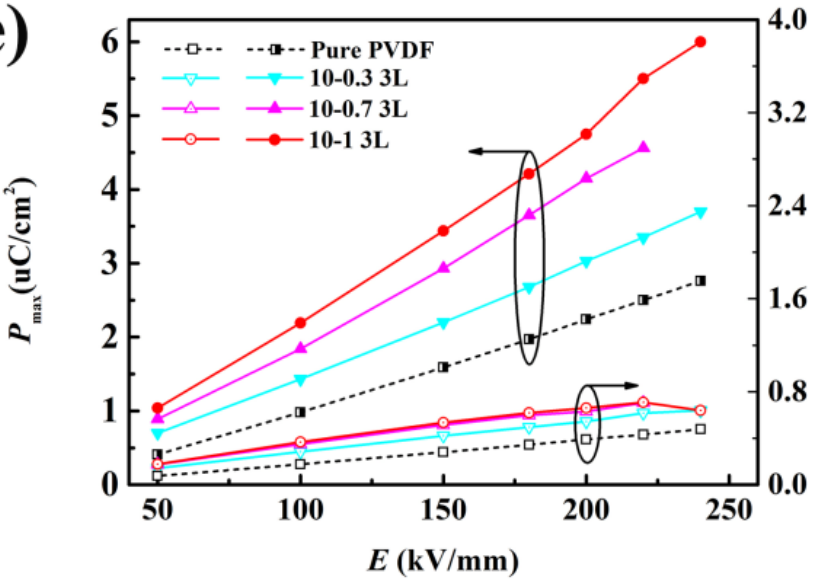

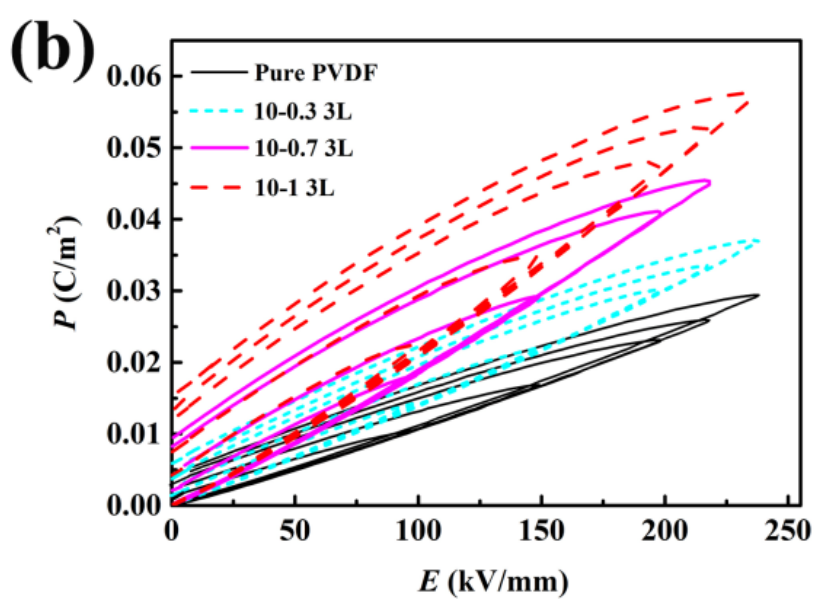

(d)
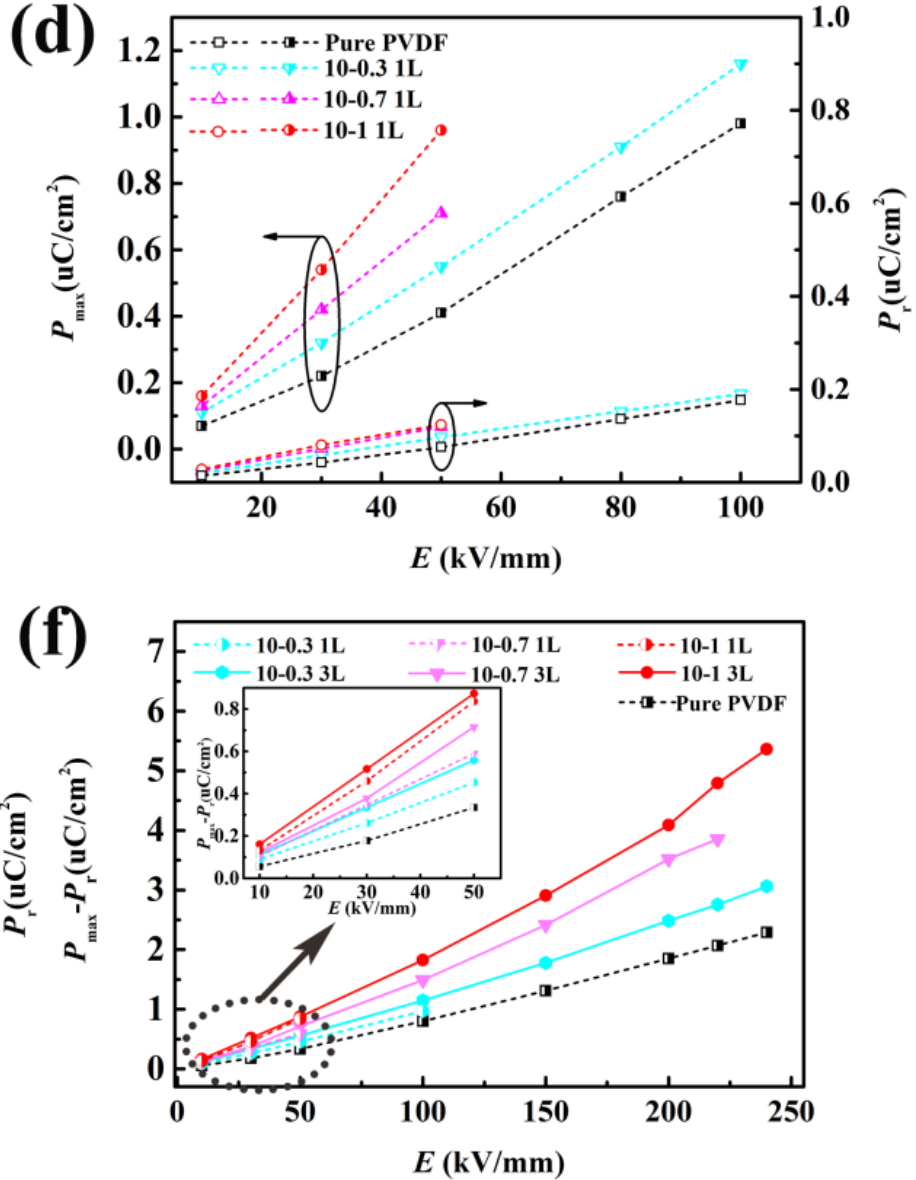

Fig. $6 P$ - $E$ loops of (a) single-layer and (b) trilayer composites under varied electric fields; (c) $P-E$ loops of single-layer and trilayer composites under $50 \mathrm{kV} / \mathrm{mm}$; The variation of maximum displacement $P_{\max }$ and remnant displacement $P_{\mathrm{r}}$ of the (d) single layer and (e) trilayer composites with different external electric fields; (f) $\left(P_{\max }-P_{\mathrm{r}}\right)$ of the single layer and trilayer composites with different external electric fields.

The frequency dependences of dielectric permittivity and matrix. ${ }^{[46-49]}$ In addition, the dielectric performances of the loss tangent for the hybrid single-layer and trilayer composites are presented in Fig. 3. As shown in Fig. 3a, the ternary singlelayer composites show obviously improved dielectric permittivity in comparison with pure P(VDF-HFP), the dielectric permittivity increases with increasing the loading of TiN nanoparticles, which is due to the interfacial polarization and the formation of equivalent microcapacitors occurred at the interfaces between the nanofillers and P(VDF-HFP) single-layer composites filled with only one type of fillers are also obtained and presented in Fig. S2. We can see that, although the dielectric permittivity of the TiN/P(VDF-HFP) composites can reach as high as $~ 50 @ 10 \mathrm{kHz}$, the loss tangent also exceeds $5 @ 10 \mathrm{kHz}$, which is not suitable for practical applications. On the contrary, although the low loss tangents are well maintained in the BT/P(VDF-HFP) composites, the enhancement of dielectric permittivity (i.e., $\varepsilon_{\mathrm{r}}$ 
$\sim 13 @ 10 \mathrm{kHz})$ is limited. Therefore, the ternary single-layer composites co-filled with $\mathrm{BaTiO}_{3}$ and TiN nanoparticles exhibit superior comprehensive dielectric performances over the single-layer composites filled with only one type of fillers. It is worth noting that the trilayer composites exhibit further higher dielectric permittivity compared with the ternary single-layer composites in Fig. 3c. This phenomenon could be attributed to the extra charge carrier accumulation and interfacial polarization at the interfaces between adjacent layers. ${ }^{[50,51]}$

Besides, the obvious enhancement of dielectric permittivity is achieved without sacrificing low loss. As shown in Fig. $3 \mathrm{~b}$ and $3 \mathrm{~d}$, the loss tangent of the trilayer composites are significantly lower than that of the single-layer ternary composites, even close to that of pure P(VDF-HFP). In the layer-structured composites, the pure P(VDF-HFP) layer can effectively hamper the formation of leakage conduction path throughout the whole composites, yielding the suppressed conductivities (Fig. S3) and thus retain the low loss well. Moreover, the layer-structured composites exhibit similar permittivity frequency dispersion behaviors with pure $\mathrm{P}(\mathrm{VDF}-\mathrm{HFP})$, that is, the permittivity decreases slightly with increasing frequency owing to the gradual dielectric relaxation of different types of polarizations. The weak frequency dependence of permittivity is beneficial to the application in a wide frequency range. Fig. 3(e-f) shows the comparison of dielectric performance at $10 \mathrm{kHz}$ between the ternary singlelayer and trilayer composites with the same content of $\mathrm{BaTiO}_{3}$ and TiN. It can be observed that the dielectric properties of trilayer composites are always better than those of the ternary single-layer composites with the same composition, that is, the dielectric permittivity is higher, at the same time, dielectric loss is lower. The trilayer composite 10-1 displays a dielectric permittivity of $\sim 21.5 @ 10 \mathrm{kHz}$, which is about 2.9 and 1.5 times of the pure $\mathrm{P}(\mathrm{VDF}-\mathrm{HFP})\left(\varepsilon_{\mathrm{r}} \sim 7.5 @ 10 \mathrm{kHz}\right)$ and its ternary single-layer counterpart $\left(\varepsilon_{\mathrm{r}} \sim 14.4 @ 10 \mathrm{kHz}\right)$ Moreover, the dielectric permittivity of the trilayer composites is further optimized by increasing the $\mathrm{BaTiO}_{3}$ content to 15 or $20 \mathrm{vol} \%$ (Fig. S4a and S5a). It can be concluded that constructing layered structures is an effective way to achieve enhanced dielectric permittivity without sacrificing low loss.

It should be noted that the breakdown strengths of dielectric materials are of great significance in practical applications. Specifically, when the dielectric materials are applied in film capacitors, a higher breakdown strength allows a larger charging electric field and full polarization, hence a higher energy-storage density. Therefore, the breakdown strengths of the composites are further evaluated using the two-parameter Weibull distribution, which is described by Eq (1):

$$
\mathrm{C}\left(E_{t}\right)=1-\exp \left[-\left(E_{t} / \alpha\right)^{\beta}\right]
$$

where $\mathrm{C}\left(E_{t}\right)$ is the cumulative failure probability, $E_{t}$ is the experimentally tested breakdown electric field, $\alpha$ is the electric field, for which there is a $63.2 \%$ probability of sample breakdown (Weibull breakdown strength, $E_{\mathrm{b}}$ ), and $\beta$ is a shape parameter or the slope of the derived logarithm function reflecting the scatter of the tested $E_{\mathrm{b}}$. When the $\beta$ value is 3, the tested data follow Gaussian distribution, and a higher value of $\beta$ implies a higher level of reliability. Fig. 4 presents the Weibull breakdown strength of the ternary single-layer and trilayer composites. As shown in Fig. 4a, all of the plots show high $\beta$ values, indicating a high reliability of the measured data Pure $\mathrm{P}(\mathrm{VDF}-\mathrm{HFP})$ has the highest $E_{\mathrm{b}}$ of $\sim 368.82 \mathrm{kV} / \mathrm{mm}$. All ternary single-layer composites present low breakdown strengths mainly owing to the existence of conductive TiN nanofillers. Besides, the low breakdown strength is also attributed to the inherent low $E_{\mathrm{b}}$ of $\mathrm{BaTiO}_{3}$, the electric field distortion near $\mathrm{TiN}$ and $\mathrm{BaTiO}_{3}$ particles as well as the defects induced by the agglomeration of nanofillers. With increasing the TiN loading, the $E_{\mathrm{b}}$ of the single-layer ternary composites decreases gradually, yielding the lowest $E_{\mathrm{b}}$ of $50.24 \mathrm{kV} / \mathrm{mm}$ in the ternary single-layer 10-1 composite. Interestingly, the trilayer composites exhibit obviously an improved $E_{\mathrm{b}}$ compared with their single-layer ternary counterparts, which is also true for the trilayer composite with $20 \mathrm{vol} \% \mathrm{BaTiO}_{3}$ (Fig. S4). Especially, the $E_{\mathrm{b}}$ of trilayer 10-0.3 nanocomposite reaches $253.84 \mathrm{kV} / \mathrm{mm}$, which represents a $\sim 286 \%$ increment compared with its single-layer ternary counterpart.

The enhanced breakdown strength of trilayer composites is mainly attributed to three factors. Firstly, it is well known that the gradient distribution of electric field can be formed inside the multilayer composites when an electric field is applied. That is, the electric field tends to concentrate on the layers with low dielectric permittivity, ${ }^{[2,24,52-54]}$ therefore, the pure P(VDFHFP) layer undertakes much higher strengths of electric field than the other two layers in the trilayer composites due to the lower dielectric permittivity of P(VDF-HFP), which thus prevents the early breakdown of the whole trilayer composites because of the inherent high $E_{\mathrm{b}}$ of P(VDF-HFP). Secondly, the electric field gap between adjacent layers in the layerstructured composites could act as a barrier to hamper the development of electrical trees, leading to improved breakdown strengths..$^{[9,27,55]}$ Thirdly, the two macroscopic interfaces between the adjacent layers in the trilayer structure effectively obstruct the development of electrical trees and thus improve the $E_{\mathrm{b}}$ of the trilayer composites. Specifically, the $E_{\mathrm{b}}$ of the trilayer composites of 10-0.3, 10-0.7 and 10-1 are $\sim 286 \%, \sim 415 \%$, and $\sim 423 \%$ that of their single-layer ternary counterparts in Fig. 4b. As discussed above, when a single-layer composite is redesigned as a layer-structured composite, the macroscopic structure design and the redistribution of fillers will achieve the improvement of dielectric breakdown strengths. 

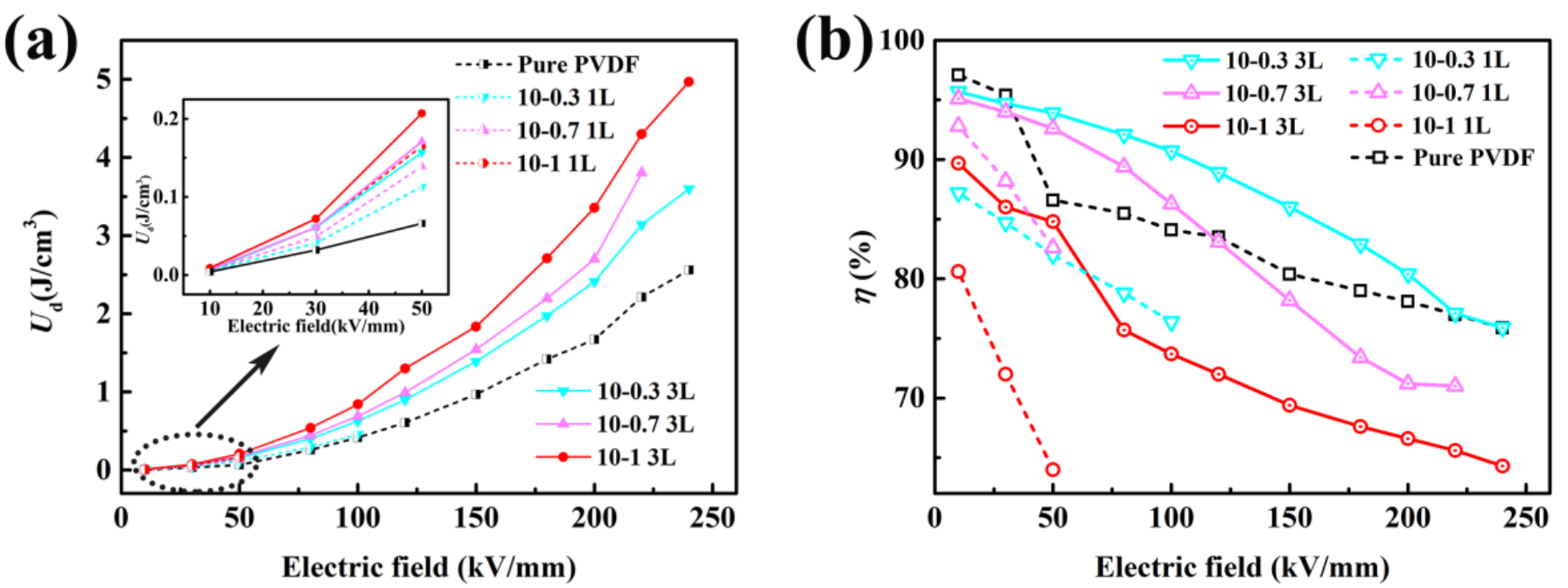

Fig. 7 (a) The discharged energy densities, and (b) discharged efficiencies of the single-layer and trilayer composites under varied external electric fields.

To better understand the remarkably enhanced $E_{\mathrm{b}}$ of the trilayer composites, finite element simulations were further performed to analyze the electric potential and electric field strength distribution in the single-layer and trilayer composites. As shown in Fig. 5(a-b), the equipotential lines of the composites are distorted around the BT particles owing to the distinct dielectric permittivity and conductivity differences between the ceramic fillers and the P(VDF-HFP) matrix. The electric field is elevated in the vertical direction of the fillers due to the electron movement driven by the electrostatic force, while it is weakened in the horizontal direction of the fillers. Obviously, the local electric field distortion phenomenon around the fillers is more severe in the single-layer ternary composite than that in the trilayer composite, as shown in Fig. $5(\mathrm{c}-\mathrm{d})$.

Moreover, the incorporation of the fillers into the outer layers leads to the electric field redistribution, where the applied electric field is mostly distributed to the middle layer and weak electric field regions are formed in the outer layers. ${ }^{[26,27]}$ In other words, the middle layer, which possesses a higher breakdown strength, undertakes a higher electric field compared with the two outer layers. Therefore, the trilayer composites could tolerate higher electric fields than singlelayer composites at the same applied electric field.

Furthermore, the distribution of current densities for the ternary single-layer and trilayer composites are also evaluated using the finite element simulation, as shown in Fig. 5(e-f). Clearly, the distribution of current density in the single-layer ternary composite is highly inhomogeneous in the vicinity of the fillers, whereas it is relatively homogeneous in the trilayer composite. In addition, the leakage current paths are observed between the adjacent fillers, which are very close to each other. For the single-layer ternary composite, the BT and TiN particles are randomly dispersed in the P(VDF-HFP) matrix. Accordingly, there may exist some regions where the BT and TiN particle are close to each other along the direction of applied electric field. Hence, the leakage current paths throughout the whole composite could be easily formed in these regions, leading to a high leakage current loss. As for the trilayer composites, the leakage current paths are restricted inside the outer layer, thus avoid the formation of leakage current paths throughout the whole composite. Consequently, the trilayer composites exhibit much lower loss and electrical conductivities than their single-layer counterparts, as displayed in Fig. 3 and Fig. S3. In general, the trilayer structure, in comparison with the single-layer structure, could cause the redistribution of electric field and current density, leading to the improved breakdown strengths and depressed leakage conductance loss.

The unipolar electric displacement-electric field $(P-E)$ loops for the ternary single-layer and trilayer composites are illustrated in Fig. 6(a-c), Fig. S4d and Fig. S5d. For the ternary single-layer composites, the $P-E$ loops under high electric fields were not obtained because of their aforementioned low breakdown strengths. It can be observed that, the single-layer ternary composites show much wider loops than their trilayer counterparts under the same external electric field, indicating enhanced energy loss and suppressed efficiency. Besides, the trilayer composites have higher maximum polarization $\left(P_{\max }\right)$ but lower remnant polarization $\left(P_{\mathrm{r}}\right)$ than single-layer ternary composites in Fig. 6c.

Fig. 6(d-e) show the variations of $P_{\max }$ and $P_{\mathrm{r}}$ with external electric field. It can be observed that the trilayer composites exhibit substantially enhanced $P_{\max }$ in comparison with their single-layer counterparts and pure P(VDF-HFP). Fig. 6f shows the differences of $P_{\max }$ and $P_{\mathrm{r}}$ between the ternary single-layer and trilayer composites. The $P_{\max }-P_{\mathrm{r}}$ value of the trilayer composites increases due to the obvious enhancement of $P_{\max }$ and slight elevation of $P_{\mathrm{r}}$, which is beneficial to the improvement of energy density and efficiency. For instance, the trilayer composite of 10-1 exhibits a $P_{\max }$ of $\sim 6 \mu \mathrm{C} / \mathrm{cm}^{2}$ and a $P_{\mathrm{r}}$ of $\sim 0.63 \mu \mathrm{C} / \mathrm{cm}^{2}$, while the $P_{\max }$ and $P_{\mathrm{r}}$ of pure 
$\mathrm{P}(\mathrm{VDF}-\mathrm{HFP})$ are $\sim 2.76 \mu \mathrm{C} / \mathrm{cm}^{2}$ and $\sim 0.47 \mu \mathrm{C} / \mathrm{cm}^{2}$ respectively. Consequently, the trilayer composite of 10-1 possesses a high $\left(P_{\max }-P_{\mathrm{r}}\right)$ of $5.36 \mu \mathrm{C} / \mathrm{cm}^{2}$, which is over 2 times that of pure $\mathrm{P}(\mathrm{VDF}-\mathrm{HFP})\left(P_{\max }-P_{\mathrm{r}} \sim 2.28 \mu \mathrm{C} / \mathrm{cm}^{2}\right)$. Compared with pure $\mathrm{P}(\mathrm{VDF}-\mathrm{HFP})$, the enhanced $P_{\max }$ of ternary single-layer composites is mainly attributed to the dipole polarization of nanoparticles and the interfacial polarizations at the interfaces between nanofillers and polymer matrix. As for the trilayer composites with the same composition, the contribution from the nanofillers and the interfaces is almost at the same level to their single-layer counterparts. Therefore, the obviously extra improvement of $P_{\max }$ is attributed to the interfacial polarization at the interfaces between adjacent layers for trilayer composites.

The dielectric energy-storage performances of the composites are further evaluated. The stored energy densities $\left(U_{\mathrm{s}}\right)$ of the composites were derived from the $P-E$ loops by integration of the area between the charge curve and the ordinate, while the discharged energy densities $\left(U_{\mathrm{d}}\right)$ were obtained by integrating the area between the discharge curve and the ordinate, the discharge efficiencies $\eta$ were calculated by using $\eta=U_{\mathrm{d}} / U_{\mathrm{s}}$. Fig. 7 shows the discharged energy densities and discharged efficiencies of the ternary singlelayer and trilayer composites under varied external electric fields. The single-layer ternary composites can only be charged under low electric fields because of their low breakdown strengths as aforementioned, leading to very low energy-storage densities below $0.5 \mathrm{~J} \mathrm{~cm}^{-3}$. The discharged efficiencies $\eta$ of the single-layer ternary composites decrease sharply with increasing applied electric field. For example, the $\eta$ of the single-layer ternary composites of 10-0.3, 10-0.7 and $10-1$ are decreased to $82 \%, 82.6 \%$ and $64 \%$ at $50 \mathrm{kV} / \mathrm{mm}$, respectively. On the contrary, the trilayer composites exhibit substantially enhanced energy-storage densities and high discharged efficiencies in comparison with their single-layer counterparts. The trilayer composite of 10-1 displays an energy density of $\sim 5 \mathrm{~J} \mathrm{~cm}^{-3}$, which is $180 \%$ that of pure $\mathrm{P}(\mathrm{VDF}-\mathrm{HFP})$ at $240 \mathrm{kV} / \mathrm{mm}$. Meanwhile, a satisfactory efficiency of $64.5 \%$ at $240 \mathrm{kV} / \mathrm{mm}$ is retained. This work will greatly promote the dielectric energy-storage performance optimization and the practical application of layer-structured dielectric composites in hybrid electric vehicles, medical defibrillator, and electromagnetic launch systems, etc.

\section{Conclusions}

In summary, a series of ternary $\mathrm{TiN} / \mathrm{BaTiO}_{3} / \mathrm{P}(\mathrm{VDF}-\mathrm{HFP})$ composites with different spatial distribution of fillers were prepared via solution casting and hot pressing. It is demonstrated that the layer-structured composites, in which the $\mathrm{TiN}$ and $\mathrm{BaTiO}_{3}$ nanoparticles were restricted in respective separated layers, exhibited simultaneously improved dielectric permittivity and breakdown strengths in comparison with their single-layer counterparts with randomly dispersed fillers. Particularly, the trilayer composite with 10 vol $\% \mathrm{BaTiO}_{3}$ and 1 vol\% TiN exhibited a dielectric permittivity of 21.5 at 10
$\mathrm{kHz}$ and a breakdown strength of $217 \mathrm{kV} / \mathrm{mm}$, which were $150 \%$ and $\sim 423 \%$ that of its single-layer counterpart. Consequently, a high energy density of $\sim 5 \mathrm{~J} \mathrm{~cm}^{-3}$, which was $180 \%$ that of the pure P(VDF-HFP) at $240 \mathrm{kV} / \mathrm{mm}$, were obtained along with a satisfactory efficiency of $64.5 \%$. This work shows that constructing layer-structured ternary nanocomposites with hierarchical rather than random distribution of the fillers is an effective strategy to achieve superior comprehensive dielectric energy-storage performances.

\section{Acknowledgements}

The authors acknowledge the financial support of this work by National Natural Science Foundation of China (51773187, 51402271), Foundation for Outstanding Young Scientist in Shandong Province (BS2014CL003), the "Chenguang Program" supported by the Shanghai Education Development Foundation and the Shanghai Municipal Education Commission (Grant No. 18CG56), the Innovation Program of Shanghai Municipal Education Commission (Grant No. 201901-07-00-10-E00053).

\section{Support information}

Available at https://dx.doi.org/10.30919/es8d1148.

\section{Conflict of Interest}

There are no conflicts to declare.

\section{References}

[1] Q. Li, L. Chen, M. R. Gadinski, S. H. Zhang, G. Z. Zhang, H. Y. U. Li, E. Iagodkine, A. Haque, L. Q. Chen, T. N. Jackson and Q. Wang, Nature, 2015, 523, 576-579, doi: 10.1038/nature14647.

[2]. Z. X. Sun, C. R. Ma, M. Liu, J. Cui, L. Lu, J. B. Lu, X. J. Lou, L. Jin, H. Wang and C. L. Jia, Adv. Mater, 2016, 29, 1604427 , doi: 10.1002/adma.201604427.

[3] V. P. Anju and S. K. Narayanankutty, Mater. Sci. Eng. B, 2019, 249, 114418, doi: 10.1016/j.mseb.2019.114418

[4] M. F. Guo, J. Y. Jiang, Z. H. Shen, Y. H. Lin, C. W. Nan and Y. Shen, Mater. Today, 2019, 29, 49-67, doi: 10.1016/j.mattod.2019.04.015.

[5] J. Y. Jiang, Z. H. Shen, X. K. Cai, J. F. Qian, Z. K. Dan, Y. H. Lin, B. L. Liu, C. W. Nan, L. Q. Chen and Y. Shen, Adv. Energy Mater., 2019, 9, 1803411, doi: 10.1002/aenm.201803411.

[6] H. Pan, F. Li, Y. Liu, Q. H. Zhang, M. Wang, S. Lan, Y. P. Zheng, J. Ma, L. Gu, Y. Shen, P. Yu, S. J. Zhang, L. Q. Chen, Y. H. Lin and C. W. Nan, Science, 2019, 365, 578-582, doi: 10.1126/science.aaw8109.

[7] Z. Yang, J. Wang, Y. L. Hu, C. Y. Deng, K. J. Zhu and J. H. Qiu, Compos. Pt. A., 2020, 128, 105675, doi: 10.1016/j.compositesa.2019.105675

[8] K. Han, Q. Li, C. Chanthad, M. R. Gadinski, G. Z. Zhang and Q. Wang, Adv. Funct. Mater., 2015, 25, 3505-3513, doi: 10.1002/adfm.201501070.

[9] F. H. Liu, Q. Li, J. Cui, Z. Y. Li, G. Yang, Y. Liu, L. J. Dong, C. X. Xiong, H. Wang and Q. Wang, Adv. Funct. Mater., 2017, 27 , 1606292, doi: 10.1002/adfm.201770125

[10] Z. P. Li, X. Y. Chen, C. Zheng, E. Baer, D. Langhe, M. 
Ponting, M. Brubaker, T. Hosking, R. P. Li, M. Fukuto and L. Zhu, ACS Appl. Polym. Mater., 2019, 1, 867-875, doi: 10.1021/acsami.0c18104.

[11] C. Zhang, Z. C. Shi, F. Mao, C. Q. Yang, X. T. Zhu, J. Yang, H. Zuo and R. H. Ran, ACS Appl. Mater. Interfaces, 2018, 10, 26713-26722, doi: 10.1021/acsami.8b09063.

[12] Z. C. Shi, J. Wang, F. Mao, C. Q. Yang, C. Zhang and R. H. Fan, J. Mater. Chem. A, 2017, 5, 14575, doi: 10.1039/C7TA03403B.

[13] X. Y. Huang, B. Sun, Y. K. Zhu, S. T. Li and P. K. Jiang, Prog. Mater. Sci., 2019, 100, 187-225, doi: 10.1016/j.pmatsci.2018.10.003.

[14] Z. H. Shen, J. J. Wang, Y. H. Lin, C. W. Nan, L. Q. Chen and Y. Shen, Adv. Mater., 2018, 30, 1704380, 10.1002/adma.201704380.

[15] B. H. Fan, M. Y. Zhou, C. Zhang, D. L. He and J. B. Bai, Prog. Mater. Sci., 2019, 97, 101143, doi 10.1016/j.progpolymsci.2019.06.003.

[16] X. Zhang, J. Y. Jiang, Z. H. Shen, Z. K. Dan, M. Li, Y. H. Lin, C. W. Nan, L. Q. Chen and Y. Shen, Adv. Mater., 2018, 30, 1707269, doi: 10.1002/adma.201707269.

[17] Z. C. Shi, S. G. Chen, R. H. Fan, X. A. Wang, X. Wang, Z. D. Zhang and K. Sun, J. Mater. Chem. C, 2014, 2, 6752-6757, doi: 10.1039/C4TC01117A.

[18] Z. C. Shi, R. H. Fan, K. L. Yan, K. Sun, M. Zhang, C. G. Wang, X. F. Liu and X. H. Zhang, Adv. Funct. Mater., 2013, 23, 4123-4132, doi: 10.1002/adfm.201202895.

[19] X. Huang, X. Zhang, G. K. Ren, J. Y. Jiang, Z. K. Dan, Q. H. Zhang, X. Zhang, C. W. Nan and Y. Shen, J. Mater. Chem. A, 2019, 7, 15198, doi: 10.1039/C9TA02257K.

[20] S. S. Guan, H. Li, S. G. Zhao and L. N. Guo, Compos. Sci. Technol., 2018, 158, 79-85, doi: 10.1016/j.compscitech.2017.12.038.

[21] H. C. Luo and J. Qiu, Ceram. Int., 2019, 45, 843-848, doi: 10.1016/j.ceramint.2018.09.253.

[22] C. Yang, Y. H. Lin and C. W. Nan, Carbon, 2009, 47, $1096-$ 1101, doi: 10.1016/j.carbon.2008.12.037.

[23] Y. C. Li, D. M. Zhang, S. S. Wang, Y. H. Zhan, J. Yin, X. Q. Tao, X. C. Ge, S. C. Tjong, H. Y. Liu and Y. W. Mai, Compos. Sci. Technol., 2019, 171, 152-161, doi 10.1016/j.compscitech.2018.12.022.

[24] J. Chen, Y. Li, Y. F. Wang, J. F. Dong, X. W. Xu, Q. B. Yuan, Y. J. Niu, Q. Wang and H. Wang, Compos. Sci. Technol., 2020, 186, 107912, doi: 10.1016/j.compscitech.2018.12.022.

[25] C. B. Cheng, R. H. Fan, Z. Y. Wang, Q. Shao, X. K. Guo, P. T. Xie, Y. S. Yin, Y. L. Zhang, L. Q. An, Y. H. Lei, J. E. Ryu, A. Shankar and Z. H. Guo, Carbon, 2017, 125, 103-112, doi: 10.1016/j.carbon.2017.09.037.

[26] Y. Feng, J.L. Li, W.L. Li, M.L. Li, Q.G. Chi, T.D. Zhang and W.D. Fei, Compos. Pt. A., 2019, 125, 105524, doi: 10.1016/j.compositesa.2019.105524.

[27] Y. F. Wang, J. Chen, Y. Li, Y. J. Niu, Q. Wang and H. Wang, J. Mater. Chem. A, 2019, 7, 2965, doi: 10.1039/C8TA11392K.

[28] Y. Wang, M. G. Yao, R. Ma, Q. B. Yuan, D. S. Yang, B. Cui, C. R. Ma, M. Liu and D. G. Hu, J. Mater. Chem. A, 2020, 8, $884-$
917, doi: 10.1039/C9TA11527G.

[29] B. Xie , Y. W. Zhu, M. A. Marwat, S. J. Zhang, L. Zhang and H. B. Zhang, J. Mater. Chem. A, 2018, 6, 20356-20364, doi: 10.1039/C8TA07364C.

[30] Y. L. Zhang, W. L. Li, S. C. Xu, Z. Y. Wang, W. Zhao, J. Li and W. D. Fei, J. Mater. Chem. A, 2018, 6, 24550, doi: 10.1039/C8TA09396B

[31] W. H. Yang, S. H. Yu, R. Sun and R. X. Du, Acta Mater., 2011, 59, 5593-5602, doi: 10.1016/j.actamat.2011.05.034.

[32] J. W. Lee and J. H. Koh, Ceram. Int., 2017, 43, 9493-9497, doi: 10.1016/j.ceramint.2017.04.130.

[33] Y. C. Ding, Q. Wu, D. Zhao, W. Ye, M. Hanif and H. Q. Hou, Eur. Polym. J., 2013, 49, 2567-2571, doi: 10.1016/j.eurpolymj.2013.05.016.

[34] B. H. Fan, J. W. Zha, D. R. Wang, J. Zhao, Z. F. Zhang and Z. M. Dang, Compos. Sci. Technol., 2013, 80, 66-72. doi: 10.1016/j.compscitech.2013.02.021.

[35] W. H. Xu, Y. C. Ding, S. H. Jiang, W. Ye, X. J. Liao and H. Q. Hou, Polym. Compos., 2016, 37, 794-801, doi: 10.1002/pc.23236.

[36] H. Y. Wang, Q. Fu, J. Q. Luo, D. M. Zhao, L. H. Luo and W. P. Li, Appl. Phys. Lett., 2017, 110, 242902, doi: 10.1063/1.4986443.

[37] J. Z. Hu, L. Zhang, Z. M. Dang and D. R. Wang, Compos. Sci. Technol., 2017, 148, 20-26, doi: 10.1016/j.compscitech.2017.05.009.

[38] P. W. Jaschin, R. Bhimireddi and K. B. Varma, ACS Appl. Mater. Interfaces, 2018, 10, 27278-27286, doi: 10.1021/acsami.8b07786.

[39] W. Y. Li, Z. Q. Song, J. Qian, Z. Y. Tan, H. Y. Chu, X. Y. Wu and W. Nie, Compos. Sci. Technol., 2018, 163, 71-80, doi: 10.1016/j.compscitech.2018.05.008.

[40] Y. F. Feng, Q. H. Deng, C. Peng and Q. Wu, Ceram Int., 2019, 45, 7923-7930, doi: 10.1016/j.ceramint.2019.01.104.

[41] Z. B. Pan, S. Xing, H. T. Jiang, J. J. Liu, S. W. Huang and J. W. Zhai, J. Mater. Chem. A, 2019, 7, 15347, doi: 10.1039/C9TA03292D.

[42] G. F. Liu, Y. Chen, M. J. Gong, X. Y. Liu, Z. K. Cui, Q. B. Pei, J. L. Gu, C. Huang and Q. X. Zhuang, J. Mater. Chem. C, 2018, 6, 10829-10837, doi: 10.1039/C8TC03868F.

[43] A. Zyane, F. Brouillette, A. Belfkira, R. Lucas and P. Marchet, J. Appl. Polym. Sci., 2018, 135, 46147, doi: 10.1002/app.46147.

[44] J. Wang J, Z. C. Shi, F. Man, X. Wang, K. Zhang and J. Shi, RSC Adv., 2016, 6, 43429, doi: 10.1039/C6RA06157E.

[45] M. Z. Zhao, Q. Fu, Y. F. Hou, L. H. Luo and W. P. Li, ACS Omega, 2019, 4, 1000-1006, doi: 10.1021/acsomega.9b00567.

[46] X. T. Zhu, J. Yang, D. Dastan, H. Garmestani, R. H. Fan and Z. C. Shi, Compos. Pt. A., 2019, 125, 105521, doi: 10.1016/j.compositesa.2019.105521.

[47] Z. Y. Wang, K. Sun, P. T. Xie, Q. Hou, Y. Liu, Q. L. Gu and R. H. Fan, Acta Mater., 2020, 185, 412-419, doi: 10.1016/j.actamat.2019.12.034

[48] P. T. Xie, Y. F. Li, Q. Hou, K. Y. Sui, C. Z. Liu, X. Y. Fu, J. X. Zhang, V. Murugadoss, J. C. Fan, Y. P. Wang, R. H. Fan and Z. 
H. Guo, J. Mater. Chem. C, 2020, 8, 3029-3039, doi: 10.1039/C9TC06378A.

[49] J. Yang, X. T. Zhu, H. L. Wang, X. Wang, C. C. Hao, R. H. Fan, D. Dastan and Z. C. Shi, Compos. Pt. A., 2020, 131, 105814, doi: 10.1039/C9TC06378A.

[50] C. Zhang, Z. C. Shi, F. Mao, C. Q. Yang, J. Yang, X. T. Zhu and H. Zuo, J. Mater. Sci., 2018, 53, 1-13,

[51] J. Wang, Z. C. Shi, F. Mao, S. G. Chen and X. Wang, ACS Appl. Mater. Interfaces, 2017, 9, 1793-1800, doi: 10.1021/acsami.6b12786.

[52] Y. Zeng, Z. H. Shen, Y. Shen, Y. H. Lin and C. W. Nan, Appl. Phys. Lett., 2018, 112, 103902, doi: 10.1063/1.5012006.

[53] J. Y. Pei, J. W. Zha, W. Y. Zhou, S. J. Wang, S. L. Zhong, L. J. Yin, M. S. Zheng, H. W. Cai and Z. M. Dang, Appl. Phys. Lett., 2019, 114, 103702, doi: 10.1063/1.5088085.

[54] J. Y. Jiang, Z. H. Shen, J. F. Qian, Z. K. Dan, M. F. Guo, Y. He, Y. H. Lin, C. W. Nan, L. Q. Chen and Y. Shen, Nano Energy, 2019, 62, 220-229, doi: 10.1016/j.nanoen.2019.05.038.

[55] J. Y. Jiang, Z. H. Shen, J. F. Qian, Z. K. Dan, M. F. Guo, Y. H. Lin, C. W. Nan, L. Q. Chen and Y. Shen, Energy Storage Mater., 2019, 18, 213-221, doi: 10.1016/j.ensm.2018.09.013.

\section{Author information}

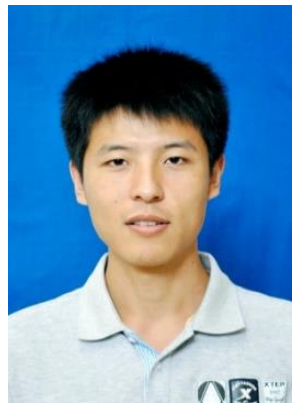

inorganic/organic

metamaterials, etc. He has authored and/or co-authored more than 40 papers in the peer-reviewed journals.

Dr. Zhicheng Shi is currently an Associate Professor at School of Materials Science and Engineering, Ocean University of China. He received his Ph.D. degree of Materials Science from Shandong University in 2013. He worked in Georgia Institute of Technology as a visiting scholar in 2017 and 2018. His current research interests include dielectric materials, nanocomposites, electromagnetic

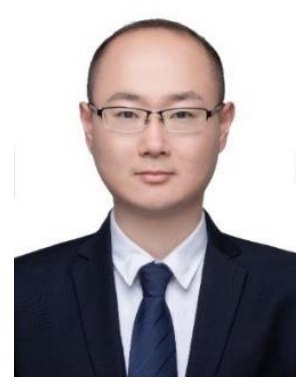

Dr. Peitao Xie obtained his bachelor and doctoral degree at Shandong University in 2014 and 2019, respectively. Now, he works for School of Material Science and Engineering, Qingdao University. His research interests include metamaterials, dielectric materials, magnetic materials and so on.

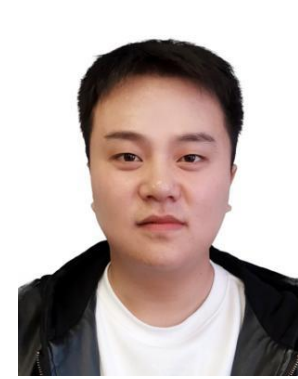

Liang Sun is a graduate student at College of Materials Science and Engineering, Ocean University of China. His current research focuses on dielectric energy storage polymer composites.
Publisher's Note: Engineered Science Publisher remains neutral with regard to jurisdictional claims in published maps and institutional affiliations. 